\title{
GLOBAL SCHAUDER DECOMPOSITIONS OF LOCALLY CONVEX SPACES
}

\author{
MILENA VENKOVA*
}

\begin{abstract}
We define global Schauder decompositions of locally convex spaces and prove a necessary and sufficient condition for two spaces with global Schauder decompositions to be isomorphic. These results are applied to spaces of entire functions on a locally convex space.
\end{abstract}

Given two spaces, $E$ and $F$, with Schauder (or even $\mathscr{S}$-absolute) decompositions, the existence of isomorphisms between the spaces forming the decompositions does not imply that $E$ and $F$ are isomorphic. In order to tackle this problem when the underlying decompositions consist of Banach spaces, P. Galindo, M. Maestre and P. Rueda defined in [12] a subclass of $\mathscr{S}$-absolute decompositions of Fréchet spaces: R-Schauder decompositions. To consider the corresponding problem when $E$ and $F$ are locally convex spaces and the underlying decompositions are not necessarily Banach spaces, we were led to define global Schauder decompositions.

\section{Introduction}

In this section we give initial definitions and preliminary results.

First we introduce notation that will be used throughout the article. Let $E$ denote a locally convex space over the complex numbers $C$, and let $E^{\prime}$ denote the space of all continuous linear functionals on $E$. When $E^{\prime}$ is endowed with the strong topology (i.e. the topology of uniform convergence over the bounded subsets of $E$ ), we denote it by $E_{\beta}^{\prime}$.

For $E$ a locally convex space we let $\mathscr{P}\left({ }^{n} E\right)$ denote the space of all continuous $n$-homogeneous polynomials on $E$. The topology on $\mathscr{P}\left({ }^{n} E\right)$ of uniform convergence over the compact (respectively bounded) subsets of $E$ is denoted by $\tau_{0}$ (respectively $\tau_{b}$ ). A third topology on $\mathscr{P}\left({ }^{n} E\right)$ can be defined in the following way. A semi-norm $p$ on $\mathscr{P}\left({ }^{n} E\right)$ is $\tau_{w}$-continuous if for every zero

\footnotetext{
* Supported by a Basic Research Grant of Science Foundation Ireland.

Received June 9, 2005.
} 
neighbourhood $V$ in $E$ there exists a positive constant $C(V)$ such that

$$
p(P) \leq C(V)\|P\|_{V}
$$

for all $P \in \mathscr{P}\left({ }^{n} E\right)$. The topology generated by all such semi-norms is denoted by $\tau_{w}$. When $n=1, E_{i}^{\prime}:=\left(\mathscr{P}\left({ }^{1} E\right), \tau_{w}\right)$ is the inductive dual of $E$, $E_{\beta}^{\prime}:=\left(\mathscr{P}\left({ }^{1} E\right), \tau_{b}\right)$ is the strong dual of $E$ and $E_{c}^{\prime}:=\left(\mathscr{P}\left({ }^{1} E\right), \tau_{0}\right)$. By $\widehat{\bigotimes} \widehat{\otimes} E, \pi$ (respectively $\widehat{\bigotimes} E$ ) we denote the completed symmetric $n$-fold tensor product of $E$ endowed with the projective tensor topology (resp. the injective tensor topology).

For more definitions and properties of polynomials and holomorphic functions on locally convex spaces we refer the reader to [7] and [8], and for more information on locally convex spaces we refer the reader to [13] and [14].

DEFINITION 1.1. A sequence of subspaces $\left\{E_{n}\right\}_{n}$ of a locally convex space $E$ is a Schauder decomposition of $E$ if:

- For each $x$ in $E$ there exists a unique sequence of vectors $\left(x_{n}\right)_{n}, x_{n} \in E_{n}$, such that

$$
x=\sum_{n=1}^{\infty} x_{n}:=\lim _{m \rightarrow \infty} \sum_{n=1}^{m} x_{n} .
$$

- The projections $\left(u_{n}\right)_{n=1}^{\infty}$ defined by

$$
u_{m}\left(\sum_{n=1}^{\infty} x_{n}\right):=\sum_{n=1}^{m} x_{n}
$$

are continuous.

The topology on each $E_{n}$ is induced by the topology on $E$. A Schauder decomposition $\left\{E_{n}\right\}_{n}$ of a locally convex space $E$ is absolute if for each $p \in \operatorname{cs}(E)$,

$$
q\left(\sum_{n=1}^{\infty} x_{n}\right):=\sum_{n=1}^{\infty} p\left(x_{n}\right)
$$

defines a continuous semi-norm on $E$.

The following definition is our main tool in this paper.

Definition 1.2. A Schauder decomposition $\left\{E_{n}\right\}_{n=0}^{\infty}$ of a locally convex space $E$ is a global Schauder decomposition if for all $r>0$, all $x=\sum_{n=1}^{\infty} x_{n} \in$ $E$ with $x_{n} \in E_{n}$ for each $n$,

$$
r \cdot x:=\sum_{n=1}^{\infty} r^{n} x_{n} \in E ;
$$


and for each $p \in \operatorname{cs}(E)$,

$$
\operatorname{pr}\left(\sum_{n=1}^{\infty} x_{n}\right):=\sum_{n=1}^{\infty} r^{n} p\left(x_{n}\right)
$$

defines a continuous semi-norm on $E$.

In particular, taking $r=1$ we see that global Schauder decompositions are absolute.

REMARK 1.3. If $\left\{E_{n}\right\}_{n}$ is a global Schauder decomposition for the locally convex space $E$, there is a generating family of semi-norms $p \in \operatorname{cs}(E)$ of the form

$$
p\left(\sum_{n=1}^{\infty} x_{n}\right)=\sum_{n=1}^{\infty} p\left(x_{n}\right)
$$

Let $q(x):=\sup _{n} p\left(x_{n}\right)$ where $p$ is a continuous semi-norm satisfying (3). Since $\sup _{n} p\left(x_{n}\right) \leq \sum_{n=1}^{\infty} p\left(x_{n}\right)$, the semi-norm $q$ is continuous. Let $q_{2}(x):=$ $\sup _{n}\left(2^{n} p\left(x_{n}\right)\right)$, from the inequality

$q(x) \leq p(x)=\sum_{n=1}^{\infty} \frac{1}{2^{n}} 2^{n} p\left(x_{n}\right) \leq \sup _{n}\left(2^{n} p\left(x_{n}\right)\right) \sum_{n=1}^{\infty}\left(\frac{1}{2^{n}}\right)=q_{2}(x) \leq p_{2}(x)$,

it follows that the semi-norms $\left\{q(x)=\sup _{n} p\left(x_{n}\right)\right\}$ generate the topology on $E$. Moreover, condition (2) in Definition 1.2 is equivalent to the condition that for each $q \in \operatorname{cs}(E)$,

$$
q_{r}\left(\sum_{n=1}^{\infty} x_{n}\right):=\sup _{n}\left(r^{n} q\left(x_{n}\right)\right)
$$

defines a continuous semi-norm on $E$. Thus the locally convex topology of $E$ can be defined both by $l_{1}$-type or by $c_{0}$-type norms.

For completeness we will give the definitions for two other types of Schauder decompositions, $\mathscr{S}$-absolute decompositions and R-Schauder decompositions. Let $\mathscr{S}$ denote the set of all sequences $\left(\alpha_{n}\right)_{n=1}^{\infty} \subset \mathrm{C}$ such that $\lim _{\sup } \rightarrow \infty\left|\alpha_{n}\right|^{1 / n}$ $\leq 1$.

DEFINITION 1.4. A Schauder decomposition $\left\{E_{n}\right\}_{n}$ of a locally convex space $E$ is an $\mathscr{S}$-absolute decomposition if for all $\alpha=\left(\alpha_{n}\right)_{n} \in \mathscr{S}$ and $x=\sum_{n=1}^{\infty} x_{n} \in E$, with $x_{n} \in E_{n}$ for all $n$,

$$
\alpha \cdot x:=\sum_{n=1}^{\infty} \alpha_{n} x_{n} \in E
$$


and, for each $p \in \operatorname{cs}(E)$ and each $\alpha=\left(\alpha_{n}\right)_{n}$ in $\mathscr{S}$,

$$
p_{\alpha}\left(\sum_{n=1}^{\infty} x_{n}\right):=\sum_{n=1}^{\infty}\left|\alpha_{n}\right| p\left(x_{n}\right)
$$

defines a continuous semi-norm on $E$.

For results and applications of $\mathscr{S}$-absolute decompositions we refer the reader to [7] and [8]. We will just mention that a Schauder decomposition of a barrelled locally convex space satisfying (5) is an $\mathscr{S}$-absolute decomposition.

Definition 1.5. Let $\left\{E_{n}\right\}_{n}$ denote an absolute Schauder decomposition of the locally convex space $E$. We say that $\left\{E_{n}\right\}_{n}$ is a T.S. (=Taylor series) complete decomposition if for any sequence $\left(x_{n}\right)_{n}$ with $x_{n} \in E_{n}$ for all $n$, $\sum_{n=1}^{\infty} p\left(x_{n}\right)<\infty$ for all $p \in \operatorname{cs}(E)$ implies $\sum_{n=1}^{\infty} x_{n} \in E$.

T.S. completeness and conditions (1) and (5) are all completeness conditions with respect to a decomposition. In particular, the $\mathscr{S}$-absolute Schauder decomposition of a sequentially complete locally convex space is T.S. complete. If $\left\{E_{n}\right\}_{n}$ is a T.S. complete and global Schauder decomposition, then it is an $\mathscr{S}$-absolute decomposition.

Let us now consider the case when $E$ is a Fréchet space such that there is a sequence of Banach spaces $\left\{E_{n}\right\}_{n=0}^{\infty}$ which is a Schauder decomposition of $E$. Let $0<R \leq \infty$. The decomposition $\left\{E_{n}\right\}_{n=0}^{\infty}$ is $R$-Schauder ([12]) if for every sequence $\left(x_{n}\right)_{n}, x_{n} \in E_{n}$, the series $x=\sum_{n=1}^{\infty} x_{n}$ converges in $E$ if and only if $\lim \sup _{n}\left\|x_{n}\right\|_{n}^{1 / n} \leq 1 / R$.

If $E$ is a Fréchet space and $\left\{E_{n}\right\}_{n=0}^{\infty}$ is an $\infty$-Schauder decomposition of $E$ consisting of Banach spaces, then it is a global Schauder decomposition of $E$. Indeed, let $A=\left\{\left(r^{n}\right)_{n}: r>0\right\}$, consider the Köthe sequence space $\lambda^{1}\left(A,\left(E_{n}\right)_{n}\right)$. This is the Fréchet space $\left\{\left(x_{n}\right)_{n} \in \prod_{n=1}^{\infty} E_{n}: p_{r}\left(\sum_{n=0}^{\infty} x_{n}\right):=\right.$ $\sum_{n=0}^{\infty} r^{n}\left\|x_{n}\right\|_{n}<\infty$ for all $\left.r>0\right\}$, endowed with the topology generated by the semi-norms $\left\{p_{r}\right\}_{r>0}$. Clearly, $\left\{E_{n}\right\}_{n=0}^{\infty}$ forms a global Schauder decomposition of $\lambda^{1}\left(A,\left(E_{n}\right)_{n}\right)$. By ([12], Theorem 1) $E$ is topologically isomorphic to $\lambda^{1}\left(A,\left(E_{n}\right)_{n}\right)$, hence $\left\{E_{n}\right\}_{n=0}^{\infty}$ forms a global Schauder decomposition of $E$.

To show that the converse is not true, consider the Köthe matrix $A^{*}=$ $\left\{\left((n r)^{n}\right)_{n}: r>0\right\}$ and a sequence of Banach spaces $\left\{E_{n}\right\}_{n=0}^{\infty}$. The corresponding Köthe sequence space $\lambda^{1}\left(A^{*},\left(E_{n}\right)_{n}\right)=\left\{\left(x_{n}\right)_{n} \in \prod_{n=1}^{\infty} E_{n}\right.$ : $p_{r}^{*}\left(\sum_{n=0}^{\infty} x_{n}\right):=\sum_{n=0}^{\infty}(n r)^{n}\left\|x_{n}\right\|_{n}<\infty$ for all $\left.r>0\right\}$ endowed with the topology generated by the semi-norms $\left\{p_{r}^{*}\right\}_{r>0}$ is a Fréchet space. It is easy to check that $\left\{E_{n}\right\}_{n=0}^{\infty}$ is a global Schauder decomposition of $\lambda^{1}\left(A^{*},\left(E_{n}\right)_{n}\right)$. Let $x=\sum_{n=1}^{\infty} x_{n} \in \prod_{n=1}^{\infty} E_{n}$ such that $\left\|x_{n}\right\|_{n}=1 / n^{n}$, then $\lim \sup _{n}\left\|x_{n}\right\|_{n}^{1 / n}=0$. On the other hand, for $r \geq 1$ the series $p_{r}(x)=\sum_{n=0}^{\infty}(n r)^{n}\left\|x_{n}\right\|_{n}$ is divergent 
hence $x$ does not belong to $\lambda^{1}\left(A^{*},\left(E_{n}\right)_{n}\right)$, i.e. $\left\{E_{n}\right\}_{n=0}^{\infty}$ is not an $\infty$-Schauder decomposition for $\lambda^{1}\left(A^{*},\left(E_{n}\right)_{n}\right)$.

\section{Application of Global Schauder Decompositions}

Proposition 2.1. Let $E$ and $F$ be locally convex spaces. Let $\left\{E_{n}\right\}_{n=0}^{\infty}$ and $\left\{F_{n}\right\}_{n=0}^{\infty}$ be T.S.-complete global Schauder decompositions for $E$ and $F$ respectively. For each $n$ let

$$
T_{n}: E_{n} \longrightarrow F_{n}
$$

be an isomorphism satisfying the following two conditions:

(A) For every $q \in \operatorname{cs}(F)$ there exist $p \in \operatorname{cs}(E)$ and positive numbers $c$ and t such that

$$
q\left(T_{n}\left(x_{n}\right)\right) \leq c t^{n} p\left(x_{n}\right)
$$

for every $x=\sum_{n=0}^{\infty} x_{n}$ in $E$ and every positive integer $n$.

(B) For every $p \in \operatorname{cs}(E)$ there exist $q \in \operatorname{cs}(F)$ and positive numbers $d$ and $v$ such that

$$
p\left(T_{n}^{-1}\left(y_{n}\right)\right) \leq d v^{n} q\left(y_{n}\right)
$$

for every $y=\sum_{n=0}^{\infty} y_{n}$ in $F$ and every positive integer $n$.

Then $T=\sum_{n=0}^{\infty} T_{n}$ is an isomorphism between $E$ and $F$.

Proof. Let $q \in \operatorname{cs}(F)$. By condition (A) there exist $p \in \operatorname{cs}(E)$ and positive numbers $c$ and $t$ such that

$$
\sum_{n=0}^{\infty} q\left(T_{n}\left(x_{n}\right)\right) \leq c \sum_{n=0}^{\infty} t^{n} p\left(x_{n}\right)=c p_{t}(x)<\infty
$$

for every $x=\sum_{n=0}^{\infty} x_{n} \in E$. Thus $T$ is well defined and continuous. Let $y=$ $\sum_{n=0}^{\infty} y_{n} \in F$, we will prove that $T$ is surjective. Since $T_{n}$ is an isomorphism for every $n$, there exist $\left\{x_{n}\right\}_{n}, x_{n} \in E_{n}$, such that $T\left(x_{n}\right)=y_{n}$. Let $p \in \operatorname{cs}(E)$. By condition (B) there exist $q \in \operatorname{cs}(F)$ and positive numbers $d$ and $v$ such that

$$
\sum_{n=0}^{\infty} p\left(x_{n}\right)=\sum_{n=0}^{\infty} p\left(T_{n}^{-1}\left(y_{n}\right)\right) \leq d \sum_{n=0}^{\infty} v^{n} q\left(y_{n}\right)=d q_{v}(y)<\infty .
$$

Since $\left\{E_{n}\right\}_{n=0}^{\infty}$ is T.S. complete, $x=\sum_{n=0}^{\infty} x_{n} \in E$ and $T(x)=y$.

Define $S=\sum_{n=0}^{\infty} T_{n}^{-1}$. Since the hypotheses are symmetric with respect to $E$ and $F$, the above also proves that $S$ is well defined and continuous. It is easy to check that $S$ is the inverse of $T$.

The converse proposition holds in a more general situation. 
Proposition 2.2. Let $E$ and $F$ be locally convex spaces, and let $\left\{E_{n}\right\}_{n=0}^{\infty}$ and $\left\{F_{n}\right\}_{n=0}^{\infty}$ be their respective Schauder decompositions. Let

$$
T: E \longrightarrow F
$$

be an isomorphism such that $T\left(E_{m}\right) \subseteq F_{m}$ for every positive integer $m$. Then $T_{m}:=\left.T\right|_{E_{m}} \longrightarrow F_{m}$ is an isomorphism for each $m$ and $T_{m}$ satisfies conditions (A) and (B) of Proposition 2.1.

Proof. Let $y_{m} \in F_{m} \subset F$. Since $T$ is surjective there exists $x=\sum_{n=0}^{\infty} x_{n}$ such that $T(x)=\sum_{n=0}^{\infty} T\left(x_{n}\right)=y_{m}$. By hypothesis $T\left(x_{n}\right) \in F_{n}$ for every $n$, hence $T_{m}\left(x_{n}\right)=0$ for $m \neq n$ and $y_{m}=T\left(x_{m}\right)=T_{m}\left(x_{m}\right)$, i.e. $T_{m}$ is surjective. Since $T$ is injective $T_{m}$ is also injective. Thus $T_{m}$ is a bijective mapping. The continuity of $T_{m}$ and $T_{m}^{-1}$ follows from the continuity of $T$ and $T^{-1}$.

We now show that conditions (A) and (B) are satisfied. Let $q \in \operatorname{cs}(F)$. Since $T$ is continuous, there exist $p \in \operatorname{cs}(E)$ and $c>0$ such that $q(T(x)) \leq c p(x)$ for every $x \in E$. In particular, for $x=x_{m} \in E_{m}$ we have

$$
q_{m}\left(T_{m}\left(x_{m}\right)\right) \leq c p_{m}\left(x_{m}\right) .
$$

Hence inequality (7) is satisfied for $t=1$. Condition (B) follows in a similar way from the continuity of $T^{-1}$.

\section{Stability Properties of Global Schauder Decompositions}

The following lemma is an adjustment of ([8], Lemma 3.31).

Lemma 3.1. Let $E$ be a barrelled locally convex space and $\left\{E_{n}\right\}_{n=0}^{\infty}$ be a Schauder decomposition of $E$ satisfying condition (1), then $\left\{E_{n}\right\}_{n=0}^{\infty}$ is a global Schauder decomposition of $E$.

Proof. Let $p$ be a continuous semi-norm on $E$, and let $r>0$. The set

$$
\begin{aligned}
\left\{x \in E: p_{r}(x)=\sum_{n=1}^{\infty} r^{n} p\left(x_{n}\right) \leq\right. & 1\}_{\infty}^{\infty}\left\{x=\sum_{m=1}^{\infty} x_{n} \in E: \sum_{i=1}^{m} r^{i} p\left(x_{i}\right) \leq 1\right\} \\
& =\bigcap_{n=1}^{\infty}\{
\end{aligned}
$$

is a barrel, and consequently a neighbourhood of zero in $E$. Thus $p_{r}$ is continuous for every $r>0$.

Lemma 3.2. Let $E$ be a sequentially complete locally convex space and $\left\{E_{n}\right\}_{n=0}^{\infty}$ be a Schauder decomposition of $E$ satisfying condition (2), then $\left\{E_{n}\right\}_{n=0}^{\infty}$ is a global Schauder decomposition of $E$. 
Proof. Let $x=\sum_{n=1}^{\infty} x_{n} \in E$ and $r>0$, denote $s_{n}:=\sum_{i=1}^{n} r^{i} x_{i}$. If $p \in \operatorname{cs}(E)$ then

$$
p\left(s_{n}-s_{m}\right)=\sum_{i=m}^{n} r^{i} p\left(x_{i}\right)=p_{r}\left(\sum_{i=m}^{n} x_{i}\right) \longrightarrow 0
$$

as $m, n \rightarrow \infty$. Thus $\left(s_{n}\right)_{n}$ is a Cauchy sequence, hence $\sum_{n=1}^{\infty} r^{i} x_{i} \in E$.

Proposition 3.3. Let $\left\{E_{n}\right\}_{n}$ denote a global Schauder decomposition for the locally convex space E. Then $\left\{\bar{E}_{n}\right\}_{n}$ is a global Schauder decomposition for the completion $\bar{E}$.

Proof. Let $x \in \bar{E}$, then there exists a net $\left(x_{\beta}\right)_{\beta} \subset E$ such that $x=$ $\lim _{\beta \rightarrow \infty} x_{\beta}$. Since $x_{\beta} \in E$ there exist $\left(x_{\beta, n}\right)_{n}$ such that $x_{\beta}=\sum_{n=0}^{\infty} x_{\beta, n}$ for every $\beta$. The nets $\left(x_{\beta, n}\right)_{\beta}$ are Cauchy for every $n$, hence there exists $x_{n} \in \bar{E}_{n}$ for every $n$ such that $x_{n}:=\lim _{\beta \rightarrow \infty} x_{\beta, n}$. Let $p \in \operatorname{cs}(E)$ be from the generating family of continuous semi-norms satisfying (3). Given $\varepsilon>0$ we can find $\beta_{0}>0$ such that

$$
\sum_{n=0}^{\infty} p\left(x_{\beta, n}-x_{\beta^{\prime}, n}\right)<\varepsilon
$$

for all $\beta, \beta^{\prime}>\beta_{0}$. By passing to the limit in $\beta^{\prime}$ and extending $p$ by continuity to the completion we get

$$
\sum_{n=0}^{\infty} p\left(x_{\beta, n}-x_{n}\right) \leq \varepsilon
$$

when $\beta>\beta_{0}$. This implies that the series $\sum_{n=0}^{\infty} x_{n}$ is convergent and $x_{\beta} \rightarrow$ $\sum_{n=0}^{\infty} x_{n}$. Since $\left(x_{\beta}\right)_{\beta}$ has a unique limit, $x=\sum_{n=0}^{\infty} x_{n}$. The projections $\left(u_{n}\right)_{n}$ defined by

$$
u_{m}\left(\sum_{n=0}^{\infty} y_{n}\right):=\sum_{n=1}^{m} y_{n}
$$

where $\sum_{n=0}^{\infty} y_{n} \in E$, are linear and continuous and hence can be extended by uniform continuity to the completion $\bar{E}$. Hence $\left\{\bar{E}_{n}\right\}_{n}$ is a Schauder decomposition for the completion $\bar{E}$.

Let $r>0$ and let $\hat{p} \in \operatorname{cs}(\bar{E})$. Since $\left\{E_{n}\right\}_{n}$ is a global Schauder decomposition for $E$, the mapping

$$
\hat{p}_{r}\left(\sum_{n=0}^{\infty} y_{n}\right)=\sum_{n=0}^{\infty} r^{n} \hat{p}\left(y_{n}\right),
$$


where $y=\sum_{n=0}^{\infty} y_{n} \in E$, defines a continuous semi-norm on $E$. Taking $r=1$ we get

$$
\begin{aligned}
\sum_{n=0}^{\infty} \hat{p}\left(x_{n}\right) & \leq \lim _{\beta \rightarrow \infty} \sum_{n=0}^{\infty} \hat{p}\left(x_{n}-x_{\beta, n}\right)+\lim _{\beta \rightarrow \infty} \sum_{n=0}^{\infty} \hat{p}\left(x_{\beta, n}\right) \\
& =\lim _{\beta \rightarrow \infty} \sum_{n=0}^{\infty} \hat{p}\left(x_{\beta, n}\right)=\lim _{\beta \rightarrow \infty} \hat{p}_{1}\left(x_{\beta}\right),
\end{aligned}
$$

hence $\sum_{n=0}^{\infty} \hat{p}\left(x_{n}\right)$ defines a continuous semi-norm on $\bar{E}$. For an arbitrary $r>0$ we have

$$
\sum_{n=0}^{\infty} r^{n} \hat{p}\left(x_{n}\right)=\sum_{n=0}^{\infty} \hat{p}\left(r^{n} x_{n}\right) \leq \lim _{\beta \rightarrow \infty} \sum_{n=0}^{\infty} \hat{p}\left(r^{n} x_{\beta, n}\right)=\lim _{\beta \rightarrow \infty} \hat{p}_{r}\left(x_{\beta}\right) .
$$

Since $\hat{p}_{r}$ is a continuous semi-norm on $E$, the limit exists and is finite. Thus $\sum_{n=0}^{\infty} r^{n} \hat{p}\left(x_{n}\right)$ defines a continuous semi-norm on $\bar{E}$. An application of Lemma 3.2 completes the proof.

Proposition 3.4. If $\left\{E_{n}\right\}_{n}$ is a global Schauder decomposition for the locally convex space $E$ then $\left\{\left(E_{n}\right)_{i}{ }^{\prime}\right\}_{n}$ is a global Schauder decomposition for the inductive dual of $E, E_{i}{ }^{\prime}$.

Proof. By ([14], 10.3) $\bar{E}_{i}^{\prime}=E_{i}^{\prime}$, and by Proposition $3.3\left\{\bar{E}_{n}\right\}_{n}$ is a global Schauder decomposition for $\bar{E}$. Hence we can assume that $E$ and all $E_{n}$ are complete.

Let $\varphi \in E^{\prime}$, we denote $\left.\varphi\right|_{E_{n}}$ by $\varphi_{n}$. By Remark 1.3 there exists a continuous semi-norm $p$ such that $|\varphi(x)| \leq p(x)$ for any $x \in E$, with $p\left(x_{n}\right) \rightarrow 0$ as $n \rightarrow \infty$, and $p(x)=\sup _{n} p\left(x_{n}\right)$ for any $x=\sum_{n=1}^{\infty} x_{n} \in E$. Hence $\varphi \in(E, p)^{\prime}$ and can be extended to $E_{p}:=\overline{(E, p) / p^{-1}(0)}$. We will denote the extension of $p$ to $E_{p}$ again by $p$, and let $\left(E_{p}\right)_{n}:=\overline{\left(E_{n},\left.p\right|_{E_{n}}\right) /\left.p\right|_{E_{n}}{ }^{-1}(0)}$. Then

$$
E_{p}=\left\{\sum_{n=1}^{\infty} x_{n}: x_{n} \in\left(E_{p}\right)_{n},\left.p\right|_{E_{n}}\left(x_{n}\right) \rightarrow 0 \text { as } n \rightarrow \infty\right\},
$$

and $p\left(\sum_{n=1}^{\infty} x_{n}\right)=\left.\sup _{n} p\right|_{E_{n}}\left(x_{n}\right)$. Let $\bar{\varphi}_{n}$ denote the extension of $\varphi_{n}$ in $\left(\left(E_{p}\right)_{n}\right)^{\prime}$. Since $\left\{E_{n}\right\}_{n}$ is a Schauder decomposition of $E, \varphi=\sum_{n=1}^{\infty} \bar{\varphi}_{n}$ pointwise on $E$. Let $p^{\prime}$ be the dual semi-norm of $p$ on $E^{\prime}$ and let $B_{p}$ be the unit ball of $E_{p}$. Then

$$
p^{\prime}\left(\varphi-\sum_{n=1}^{m} \bar{\varphi}_{n}\right)=\sup _{x \in B_{p}}\left|\varphi(x)-\sum_{n=1}^{m} \bar{\varphi}_{n}(x)\right|=\sup _{x \in B_{p}}\left|\sum_{n=m+1}^{\infty} \bar{\varphi}_{n}(x)\right| .
$$


Let $x \in E$ and $\left\{\lambda_{n}\right\}_{n} \subset \mathrm{C},\left|\lambda_{n}\right| \leq 1$ for all $n \in \mathrm{N}$. Since $\left\{E_{n}\right\}_{n}$ is an absolute decomposition and $E$ is complete, $\sum_{n=1}^{\infty} \lambda_{n} x_{n} \in E$ (see p. 189 of [8]). This allows us to choose $\left\{\lambda_{n}\right\}_{n}$ so that $\lambda_{n} \bar{\varphi}_{n}(x)=\left|\bar{\varphi}_{n}(x)\right|$ for all $n$. Since $\sup _{n}\left|\lambda_{n}\right| p\left(x_{n}\right) \leq 1$ for all $x \in B_{p}$, it follows that $\lambda \cdot x \in B_{p}$. Hence

$$
p^{\prime}\left(\varphi-\sum_{n=1}^{m} \bar{\varphi}_{n}\right)=\sup _{x \in B_{p}} \sum_{n=m+1}^{\infty}\left|\bar{\varphi}_{n}(x)\right| .
$$

Suppose $\sup _{x \in B_{p}} \sum_{n=m+1}^{\infty}\left|\bar{\varphi}_{n}(x)\right|$ does not tend to zero as $m \rightarrow \infty$. Then there exists $\delta>0$ such that for all $m \in \mathrm{N}$ we can find $x^{(m)} \in B_{p}$ with

$$
\sum_{n=m+1}^{\infty}\left|\bar{\varphi}_{n}\left(x^{(m)}\right)\right| \geq \delta .
$$

Let $m=1$ and $x^{(1)}$ be the corresponding element of $B_{p}$. There exists $m_{1}>1$ such that

$$
\sum_{n=1}^{m_{1}}\left|\bar{\varphi}_{n}\left(x_{n}^{(1)}\right)\right| \geq \frac{\delta}{2} \text {. }
$$

By induction we can build an increasing sequence $\left\{m_{j}\right\}_{j \in \mathrm{N}} \subset \mathrm{N}$ and a sequence $\left\{x^{(j)}\right\} \subset B_{p}$ such that

$$
\sum_{n=m_{j}+1}^{m_{j+1}}\left|\bar{\varphi}_{n}\left(x_{n}^{(j+1)}\right)\right| \geq \frac{\delta}{2}
$$

for all $j$. Let

$$
y_{n}= \begin{cases}0 & n \leq m_{j}, \\ \frac{1}{n} x_{n}^{(j+1)} & m_{j}+1 \leq n \leq m_{j+1}, \\ 0 & n>m_{j+1} .\end{cases}
$$

Since $p\left(x^{(j)}\right) \leq 1$ and $p(x)=\sup _{k} p\left(x_{k}\right)$, we have that $p\left(y_{n}\right) \leq 1 / n$, hence $p\left(y_{n}\right) \rightarrow 0$ as $n \rightarrow \infty$ and $\left(y_{n}\right)_{n} \subset B_{p}$. This implies that $\sum_{n=1}^{\infty} y_{n} \in E_{p}$. As before we can choose $\left\{\lambda_{n}\right\}_{n} \subset \mathrm{C},\left|\lambda_{n}\right| \leq 1$ for all $n \in \mathrm{N}$, so that $\varphi\left(\sum_{n=1}^{\infty} \lambda_{n} y_{n}\right)=\sum_{n=1}^{\infty}\left|\varphi\left(y_{n}\right)\right|$. However

$$
\sum_{n=1}^{\infty}\left|\varphi\left(y_{n}\right)\right|=\sum_{n=1}^{\infty} \frac{1}{n}\left|\bar{\varphi}_{n}\left(x_{n}^{(j+1)}\right)\right| \geq \frac{\delta}{2} \sum_{n=1}^{\infty} \frac{1}{n},
$$

i.e. $\sum_{n=1}^{\infty}\left|\varphi\left(y_{n}\right)\right|$ is divergent, a contradiction. Hence $p^{\prime}\left(\varphi-\sum_{n=1}^{m} \bar{\varphi}_{n}\right) \rightarrow 0$ and $\varphi=\sum_{n=1}^{\infty} \varphi_{n} \in E_{p}^{\prime}$. Since, by definition, $E_{i}{ }^{\prime}=\operatorname{ind}_{p \in \operatorname{cs}(E)}\left(\overline{(E, p) / p^{-1}(0)}\right)^{\prime}$, 
the mapping $E_{p}^{\prime} \longrightarrow E_{i}^{\prime}$ is continuous, and $\varphi=\sum_{n=1}^{\infty} \varphi_{n}$ in $E_{i}^{\prime}$. Moreover, by ([14], Proposition 10.3.4) the canonical surjection $E_{i}{ }^{\prime} \longrightarrow\left(E_{n}\right)_{i}^{\prime}$ is open and continuous, hence $E_{i}^{\prime}$ induces the inductive topology on $\left(E_{n}\right)^{\prime}$. Thus $\left\{\left(E_{n}\right)_{i}{ }^{\prime}\right\}_{n}$ is a Schauder decomposition for $E_{i}{ }^{\prime}$. The above also shows that $\varphi_{n}(x)=$ $\varphi_{n}\left(x_{n}\right)=\varphi\left(x_{n}\right)$.

Next we show that $\left\{\left(E_{n}\right)_{i}{ }^{\prime}\right\}_{n}$ is a global Schauder decomposition for $E_{i}{ }^{\prime}$. Let $\varphi \in E_{i}^{\prime}, \varphi=\sum_{n=1}^{\infty} \varphi_{n}$, and let $r>0$. If $x \in E$ then $r \cdot x \in E$ and

$$
(r \cdot \varphi)(x):=\sum_{n=1}^{\infty} r^{n} \varphi_{n}\left(x_{n}\right)=\varphi\left(\sum_{n=1}^{\infty} r^{n} x_{n}\right)=\varphi(r \cdot x)
$$

is well defined. Since $\varphi$ is continuous there exists a continuous semi-norm $p$ on $E$ such that $|\varphi(x)| \leq p(x)$ for any $x \in E$. Then

$$
|(r \cdot \varphi)(x)| \leq \sum_{n=1}^{\infty} r^{n} \varphi_{n}\left(x_{n}\right) \leq p_{r}(x) .
$$

Since $p_{r}$ is a continuous semi-norm on $E$, this implies that $r \cdot \varphi \in E^{\prime}$. An application of Lemma 3.1 completes the proof.

A proof for the following proposition can be obtained by modifying the proof of Proposition 3.4.

Proposition 3.5. If $\left\{E_{n}\right\}_{n}$ is an $\mathscr{S}$-absolute decomposition for the locally convex space $E$ then $\left\{\left(E_{n}\right)_{i}^{\prime}\right\}_{n}$ is an $\mathscr{S}$-absolute decomposition for $E_{i}{ }^{\prime}$.

Next we look at the strong dual of a locally convex space.

Proposition 3.6. If $\left\{E_{n}\right\}_{n}$ is a global Schauder decomposition for the locally convex space $E$ then $\left\{\left(E_{n}\right)_{\beta}^{\prime}\right\}_{n}$ is a global Schauder decomposition for $E_{\beta}^{\prime}$.

Proof. Let $\varphi=\sum_{n=0}^{\infty} \varphi_{n} \in E^{\prime}$ where $\varphi_{n}:=\left.\varphi\right|_{E_{n}}$. By the continuity of $\varphi$ there exists $p \in \operatorname{cs}(E)$ such that $|\varphi(x)| \leq p(x)$ for all $x=\sum_{n=0}^{\infty} x_{n} \in E$. Let $r>0$, then

$$
\sum_{n=1}^{\infty} r^{n} \varphi_{n}\left(x_{n}\right)=\sum_{n=1}^{\infty} \varphi_{n}\left(r^{n} x_{n}\right) \leq p_{r}\left(\sum_{n=1}^{\infty} x_{n}\right) .
$$

Let $\left(\sum_{n=1}^{\infty} r^{n} \varphi_{n}\right)(x):=\lim _{n \rightarrow \infty} \sum_{i=1}^{n} r^{i} \varphi_{i}\left(x_{i}\right)$. By (8), $\sum_{n=1}^{\infty} r^{n} \varphi_{n} \in E^{\prime}$. The topology on $E_{\beta}^{\prime}$ is generated by all semi-norms of the form

$$
s(\varphi):=\sup \{|\varphi(x)|: x \in A\},
$$


for all $A$ bounded subsets of $E$. Let $B$ be a bounded set in $E, r \cdot B:=$ $\left\{\sum_{n=1}^{\infty} r^{n} x_{n}: x \in B\right\}$ and $p \in \operatorname{cs}(E)$. Then since $p_{r} \in \operatorname{cs}(E)$,

$$
\sup _{x \in r \cdot B} p(x)=\sup _{x \in B} p\left(\sum_{n=0}^{\infty} r^{n} x_{n}\right)=\sup _{x \in B} p_{r}(x)<\infty \text {. }
$$

Hence the set $r \cdot B$ is bounded in $E$. Therefore

$$
\left\|\sum_{n=m}^{\infty} r^{n} \varphi_{n}\right\|_{B}=\sup _{x \in B}\left|\sum_{n=m}^{\infty} \varphi_{n}\left(r^{n} x_{n}\right)\right|=\sup _{x \in r \cdot B}\left|\sum_{n=m}^{\infty} \varphi_{n}\right| \rightarrow 0
$$

as $m \rightarrow \infty$. Hence $\left\{\left(E_{n}\right)_{\beta}\right\}_{n}$ is a Schauder decomposition for $E_{\beta}^{\prime}$ satisfying (1). It remains to show that condition (2) is satisfied. Let $B$ be a bounded set in $E$ and let

$$
\tilde{B}:=\left\{\sum_{n=1}^{\infty} \lambda_{n} x_{n}: x \in B,\left(\lambda_{n}\right)_{n} \subset \mathrm{C} \text { such that }\left|\lambda_{n}\right| \leq 1 \text { for all } n \in \mathrm{N}\right\} .
$$

The set $\tilde{B}$ is bounded in $E$. Indeed, let $p \in \operatorname{cs}(E)$ satisfying (3). Then

$$
\sup _{x \in \tilde{B}} p(x)=\sup _{x \in B} \sum_{n=1}^{\infty} p\left(\lambda_{n} x_{n}\right)=\sup _{x \in B} \sum_{n=1}^{\infty} p\left(x_{n}\right)<\infty \text {. }
$$

This allows us to choose $\left\{\lambda_{n}\right\}_{n}$ so that $\lambda_{n} \varphi_{n}(x)=\left|\varphi_{n}(x)\right|$ for all $n$. Then

$$
\sup _{x \in 2 r \tilde{B}}|\varphi(x)|=\sup _{x \in \tilde{B}}\left|\sum_{n=1}^{\infty}(2 r)^{n} \varphi_{n}\left(x_{n}\right)\right|=\sup _{x \in B} \sum_{n=1}^{\infty}(2 r)^{n}\left|\varphi_{n}\left(x_{n}\right)\right| \geq(2 r)^{n}\left\|\varphi_{n}\right\|_{B}
$$

for all $n$. Let $q(\varphi)=\sup _{x \in B}|\varphi(x)|$, then

$$
q_{r}(\varphi) \leq \sum_{n=1}^{\infty} r^{n} \sup _{x \in B}\left|\varphi_{n}\left(x_{n}\right)\right| \leq \sum_{n=1}^{\infty} \frac{1}{2^{n}} \sup _{x \in 2 r \tilde{B}}|\varphi(x)|=\|\varphi(x)\|_{2 r \tilde{B}}
$$

is continuous on $E_{\beta}^{\prime}$.

\section{Global Schauder Decompositions of Spaces of Holomorphic Functions}

In this section $\mathscr{H}(E)$ denotes the space of entire functions on a locally convex space $E$.

Proposition 4.1. Let E be a locally convex space. Then 
(1) $\left\{\left(\mathscr{P}\left({ }^{n} E\right), \tau_{0}\right)\right\}_{n=0}^{\infty}$ is a global Schauder decomposition for $\left(\mathscr{H}(E), \tau_{0}\right)$.

(2) $\left\{\left(\mathscr{P}\left({ }^{n} E\right), \tau_{w}\right)\right\}_{n=0}^{\infty}$ is a global Schauder decomposition for $\left(\mathscr{H}(E), \tau_{\delta}\right)$ and $\left(\mathscr{H}(E), \tau_{w}\right)$.

Proof. By ([8], Proposition 3.36), $\left\{\left(\mathscr{P}\left({ }^{n} E\right), \tau_{0}\right)\right\}_{n=0}^{\infty}$ and $\left\{\left(\mathscr{P}\left({ }^{n} E\right), \tau_{w}\right)\right\}_{n=0}^{\infty}$ are $\mathscr{S}$-absolute Schauder decompositions for $\left(\mathscr{H}(E), \tau_{0}\right)$ and $\left(\mathscr{H}(E), \tau_{\delta}\right)$ respectively.

Let $f=\sum_{n=0}^{\infty} \frac{\widehat{d^{n}} f(0)}{n !} \in \mathscr{H}(E)$ and $r>0$. If $K \subset E$ is a compact balanced set, by the local boundedness of $f$ there exists a balanced open $V \subset E$ such that $K \subset V$ and $\sum_{n=0}^{\infty}\left\|\frac{\widehat{d}^{n} f(0)}{n !}\right\|_{V}<\infty$. Then

$$
\|r \cdot f\|_{1 / r V} \leq \sum_{n=0}^{\infty} r^{n}\left\|\frac{\widehat{d^{n}} f(0)}{n !}\right\|_{1 / r V}=\sum_{n=0}^{\infty}\left\|\frac{\widehat{d^{n}} f(0)}{n !}\right\|_{V} \leq\|f\|_{V} .
$$

Hence $r \cdot f \in \mathscr{H}(E)$.

Since $\left(\mathscr{H}(E), \tau_{\delta}\right)$ is barrelled, by Lemma $3.1\left\{\left(\mathscr{P}\left({ }^{n} E\right), \tau_{w}\right)\right\}_{n=0}^{\infty}$ is a global Schauder decomposition for $\left(\mathscr{H}(E), \tau_{\delta}\right)$. By replacing $V$ by $K$ in (9) we get $\|r \cdot f\|_{K} \leq\|f\|_{r K}$ for all $f \in \mathscr{H}(E)$. Hence $\left\{\left(\mathscr{P}\left({ }^{n} E\right), \tau_{0}\right)\right\}_{n=0}^{\infty}$ is a global Schauder decomposition for $\left(\mathscr{H}(E), \tau_{0}\right)$. The proof that $\left\{\left(\mathscr{P}\left({ }^{n} E\right), \tau_{w}\right)\right\}_{n=0}^{\infty}$ is a global Schauder decomposition for $\left(\mathscr{H}(E), \tau_{w}\right)$ is similar (see also Proposition 3.36 of [8]).

Propositions 4.1 and 2.1 imply

Corollary 4.2. Let $E$ be a locally convex space. Then $\tau_{\delta}=\tau_{w}$ on $\mathscr{H}(E)$ if and only iffor every $\tau_{\delta}$-continuous semi-norm $q$ there exist a $\tau_{w}$-continuous semi-norm $p$ and positive numbers $c$ and $t$ such that

$$
q\left(\frac{\widehat{d^{n}} f(0)}{n !}\right) \leq c t^{n} p\left(\frac{\widehat{d^{n}} f(0)}{n !}\right)
$$

for every $f=\sum_{n=0}^{\infty} \frac{\widehat{d^{n}} f(0)}{n !} \in \mathscr{H}(E)$ and every positive integer $n$.

Let $E$ be a locally convex space, denote

$$
\mathscr{H}_{b}(E)=\left\{f \in H(E):\|f\|_{A}<\infty \text { for every bounded set } A\right\} .
$$

The functions in $\mathscr{H}_{b}(E)$ are called holomorphic functions of bounded type. When endowed with $\tau_{b}$, the topology of uniform convergence over the bounded sets of $E, \mathscr{H}_{b}(E)$ becomes a locally convex space. The proof of Proposition 4.1 can easily be modified to show the following:

Proposition 4.3. Let $E$ be a locally convex space. Then $\left\{\left(\mathscr{P}\left({ }^{n} E\right), \tau_{b}\right)\right\}_{n=0}^{\infty}$ is a global Schauder decomposition for $\left(\mathscr{H}_{b}(E), \tau_{b}\right)$. 
In ([12], Examples 2 and 4) are given several examples of spaces of entire functions on a Banach space such that the corresponding polynomial subspaces are their $\infty$-Schauder decompositions - and, thus, their global Schauder decompositions. All our results will apply to these spaces, and in particular Proposition 2.1 reduces in this special case to ([12], Theorem 9(ii)).

Let $E$ be a Banach space with a unit ball $B_{E}$. In ([6]) the authors defined the space $\mathscr{H}_{\mathrm{bI}}(E)$ of entire functions whose restrictions to $n B_{E}$ are integral for all $n$. Endowed with the system of semi-norms $\left\{p_{n}(f)=\left\|\left.f\right|_{n B_{E}}\right\|_{I}\right\}_{n=1}^{\infty}$, $\mathscr{H}_{\mathrm{bI}}(E)$ is a Fréchet space and $\left\{\left(\mathscr{P}_{I}\left({ }^{n} E\right),\|\cdot\|_{I}\right)\right\}_{n=0}^{\infty}$ is an $\infty$-Schauder (and hence global) decomposition for $\mathscr{H}_{\mathrm{bI}}(E)$. Now consider the entire functions of bounded nuclear type on $E, H_{\mathrm{Nb}}(E)$ ([8], Definition 4.47). With the topology generated by the semi-norms $\left\{\pi_{n}(f)=\left\|\left.f\right|_{n B_{E}}\right\|_{N}\right\}_{n=1}^{\infty}, \mathscr{H}_{\mathrm{Nb}}(E)$ is a Fréchet space and a short calculation shows that $\left\{\left(\mathscr{P}_{N}\left({ }^{n} E\right),\|\cdot\|_{N}\right)\right\}_{n=0}^{\infty}$ is an $\infty$-Schauder decomposition for $\mathscr{H}_{\mathrm{Nb}}(E)$. By ([5], Theorem 2) if $\ell_{1} \hookrightarrow \widehat{\bigotimes}_{n, s, \varepsilon} E$ for some integer $n$ then $\mathscr{P}_{N}\left({ }^{n} E\right)$ and $\mathscr{P}_{I}\left({ }^{n} E\right)$ are isometrically isomorphic. By ([12], Corollary 11) we obtain

Proposition 4.4. Let E be a Banach space such that $\widehat{\bigotimes} E$ does not contain a copy of $\ell_{1}$ for any $n \in \mathrm{N}$. Then $\mathscr{H}_{\mathrm{bI}}(E)$ and $\mathscr{H}_{\mathrm{Nb}}(E)$ are isomorphic.

Furthermore, by ([5], Proposition 3) we can replace " $\widehat{\bigotimes} E$ does not contain a copy of $\ell_{1}$ for any $n \in \mathrm{N}$ " with the condition that $E^{\prime}$ has RNP.

Now let $E$ be a locally convex space, let

$$
\begin{aligned}
G_{b}(E):=\left\{\varphi \in \mathscr{H}_{b}(E)^{*}: \varphi \text { is } \tau_{0}\right. \text {-continuous } \\
\\
\left.\quad \text { on the bounded subsets of } \mathscr{H}_{b}(E)\right\} .
\end{aligned}
$$

When endowed with the topology $\tau_{g}$ of uniform convergence on the bounded subsets of $\mathscr{H}_{b}(E), G_{b}(E)$ becomes a complete locally convex space. Let $E$ be a locally convex space such that the $\tau_{b}$-bounded sets of $\mathscr{H}_{b}(E)$ are locally bounded. By ([8], Lemma 3.25) if $B$ is a locally bounded $\tau_{b}$-bounded subset of $\mathscr{H}_{b}(E)$, then it is relatively compact in $\left(\mathscr{H}_{b}(E), \tau_{0}\right)$. This allows us to apply ([15], Theorem 1.1) (see also p. 115 of [2]), and we obtain that $G_{b}(E)_{i}^{\prime}=$ $\left(\mathscr{H}_{b}(E), \tau_{b}^{\text {bor }}\right)$. We have proved the following proposition.

Proposition 4.5. Let $E$ be a locally convex space such that the $\tau_{b}$-bounded sets of $\mathscr{H}_{b}(E)$ are locally bounded. Then

$$
G_{b}(E)_{i}^{\prime}=\left(\mathscr{H}_{b}(E), \tau_{b}^{\text {bor }}\right) .
$$

If $E$ is a bornological DF space then the $\tau_{b}$-bounded sets of $\mathscr{H}_{b}(E)$ are locally bounded by ([10], Proposition 15). By ([10], Theorem 4), $\left(\mathscr{H}_{b}(E), \tau_{b}\right)$ 
is Fréchet, and hence ultrabornological, which implies $\tau_{b}=\tau_{b}^{\text {bor }}$. Thus if $E$ is a bornological DF space, $G_{b}(E)_{i}^{\prime}=\left(\mathscr{H}_{b}(E), \tau_{b}\right)$.

Proposition 4.6. Let $E$ be a locally convex space. Then the sequence $\left\{\left(\mathscr{P}\left({ }^{n} E\right), \tau_{b}^{\text {bor }}\right)^{\prime} \cap G_{b}(E)\right\}_{n=0}^{\infty}$ is a global Schauder decomposition for $G_{b}(E)$.

PRoof. The space $G_{b}(E)$ is a subspace of $\left(\mathscr{H}_{b}(E), \tau_{b}^{\text {bor }}\right)^{\prime}$ since its elements are $\tau_{b}$-continuous on the bounded sets of $\mathscr{H}_{b}(E)$ and hence are $\tau_{b}^{\text {bor }}$-continuous. Let $\left(f_{\beta}\right)_{\beta}$ be a bounded net in $H_{b}(E)$ which tends to 0 uniformly on every compact subset $K$ of $E$, and let $r>0$. Since $\left\{\left(\mathscr{P}\left({ }^{n} E\right), \tau_{b}\right)\right\}_{n=0}^{\infty}$ is a global Schauder decomposition for $\left(\mathscr{H}_{b}(E), \tau_{b}\right)$ and $r K$ is also a compact set, we have

$$
\sum_{n=0}^{\infty} r^{n}\left\|\frac{\widehat{d^{n}} f_{\beta}(0)}{n !}\right\|_{K}=\sum_{n=0}^{\infty}\left\|\frac{\widehat{d^{n}} f_{\beta}(0)}{n !}\right\|_{r K} \longrightarrow 0
$$

as $\beta \rightarrow \infty$. Hence $\left\{\sum_{n=0}^{\infty} r^{n} \frac{\widehat{d^{n}} f_{\beta}(0)}{n !}\right\}_{\beta}$ is also a bounded $\tau_{0}$-null net in $\mathscr{H}_{b}(E)$. Let $\vartheta=\sum_{n=0}^{\infty} \vartheta_{n} \in G_{b}(E)$ where $\vartheta_{n}:=\left.\vartheta\right|_{\mathscr{P}\left({ }^{n} E\right)}$. Then

$$
\left(\sum_{n=0}^{\infty} r^{n} \vartheta_{n}\right)\left(f_{\beta}\right)=\sum_{n=0}^{\infty} \vartheta_{n}\left(\sum_{n=0}^{\infty} r^{n} \frac{\widehat{d}^{n} f_{\beta}(0)}{n !}\right) \longrightarrow 0
$$

as $\beta \rightarrow \infty$. This implies $\sum_{n=0}^{\infty} r^{n} \vartheta_{n} \in G_{b}(E)$ for every $r>0$.

Now let $p$ be a $\tau_{g}$-continuous semi-norm. Without loss of generality we may assume that

$$
p(\vartheta)=\sup _{f \in B}|\vartheta(f)|,
$$

where $B$ is a bounded subset of $\mathscr{H}_{b}(E)$. Let

$$
B_{n}:=\left\{\frac{\widehat{d^{n}} f(0)}{n !}: f \in B\right\},
$$

then

$$
\begin{aligned}
\sum_{n=0}^{\infty} r^{n} p_{n}\left(\vartheta_{n}\right) & =\sum_{n=0}^{\infty} r^{n}\left\|\vartheta_{n}\left(\frac{\widehat{d^{n}} f(0)}{n !}\right)\right\|_{B_{n}}=\sum_{n=0}^{\infty}\left\|\vartheta_{n}\left(\frac{\widehat{d^{n}} f(0)}{n !}\right)\right\|_{r B_{n}} \\
& =\sup _{f \in r B}|\vartheta(f)| .
\end{aligned}
$$

Since $r B$ is also a bounded subset of $\mathscr{H}_{b}(E)$, the semi-norm $\sum_{n=0}^{\infty} r^{n} p_{n}$ is continuous. This completes the proof. 
By ([3], Proposition 1) the space of all linear functionals on $\mathscr{P}\left({ }^{n} E\right)$ which are $\tau_{0}$-continuous on its locally bounded subsets is isomorphic to $\widehat{\otimes} E$. This and Proposition 4.6 give us

COROLLARY 4.7. Let E be a locally convex space such that for every positive

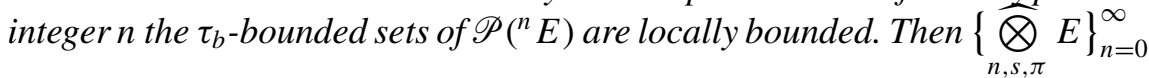
is a global Schauder decomposition for $G_{b}(E)$.

The condition in Corollary 4.7 is satisfied for example by all Fréchet spaces and all bornological DF spaces.

The following definition is given in [4].

Definition 4.8. The locally convex space $E$ is $Q$-reflexive if for every positive integer $n$ :

(1) The mapping

$$
J_{n}: \bigotimes_{n, s, \pi} E_{e}^{\prime \prime} \longrightarrow\left(\mathscr{P}\left({ }^{n} E\right), \tau_{b}\right)_{i}^{\prime}
$$

is continuous.

(2) The extension of $J_{n}$ to the completion of $\bigotimes_{e}^{\prime \prime}$ is an isomorphism between $\widehat{\bigotimes} E_{n, s, \pi} E_{e}^{\prime \prime}$ and $\overline{\left(\mathscr{P}\left({ }^{n} E\right), \tau_{b}\right)_{i}^{\prime}}$.

From Propositions 2.1 and 2.2, combined with Propositions 3.4 and 4.3 and Corollary 4.7, we obtain

Proposition 4.9. Let $E$ be a locally convex space such that the $\tau_{b}$-bounded sets of $\mathscr{H}\left(E_{\beta \beta}^{\prime \prime}\right)$ are locally bounded. If $E$ is $Q$-reflexive and $\left(J_{n}\right)_{n}$ satisfy conditions $(A)$ and $(B)$ from Proposition 2.1, then $J:=\sum_{n=0}^{\infty} J_{n}$ is an isomorphism between $G_{b}\left(E_{\beta \beta}^{\prime \prime}\right)$ and $\overline{\left(\mathscr{H}_{b}(E), \tau_{b}\right)_{i}^{\prime}}$.

We will need the following lemma.

Lemma 4.10. Let $E:=\prod_{k=1}^{\infty} F$ for some Banach space $F, E_{m}:=$ $\underbrace{F \times \cdots \times F}_{m}$ and $E^{m}:=\prod_{j=m+1}^{\infty} F$. Let $B$ be a $\tau_{b}$-bounded set in $\mathscr{H}_{b}(E)$. There exists a positive integer $n_{0}$ such that $f(x+y)=f(x)$ for all $f \in B$, $x \in E_{n_{0}}$ and $y \in E^{n_{0}}$.

Proof. Suppose our hypothesis is not true. Then for every positive integer $n$ there exist $f_{n} \in B, x_{n} \in E_{n}$ and $y_{n} \in E^{n}$ such that $f_{n}\left(x_{n}+y_{n}\right)-f_{n}\left(x_{n}\right) \neq 0$. Let $\lambda \in \mathrm{C}$, then

$$
g_{n}: \lambda \longrightarrow f_{n}\left(\lambda x_{n}+y_{n}\right)-f_{n}\left(\lambda x_{n}\right)
$$


is a non-zero entire function. For every $n$ there exists $\lambda_{n}$ such that $\left\|\lambda_{n} x_{n}\right\| \leq$ $1 / n$ and $g_{n}\left(\lambda_{n}\right) \neq 0$. Indeed, otherwise there exists a neighbourhood of zero in C such that $g_{n}$ is zero on it, and the Identity Principle implies that $g_{n}$ is identically zero on $\mathrm{C}$. Consider

$$
h_{n}: \mu \longrightarrow f_{n}\left(\lambda_{n} x_{n}+\mu y_{n}\right)-f_{n}\left(\lambda_{n} x_{n}\right) .
$$

The function $h_{n}(\mu)$ is a non-constant entire function on C, so by Liouville's Theorem is unbounded. Hence there exists $\left(\mu_{n}\right)_{n}$ in $\mathrm{C}$ such that

$$
\left|h_{n}\left(\mu_{n}\right)\right|=\left|f_{n}\left(\lambda_{n} x_{n}+\mu_{n} y_{n}\right)-f_{n}\left(\lambda_{n} x_{n}\right)\right|>n+\left|f_{n}\left(\lambda_{n} x_{n}\right)\right|
$$

for every $n$. Then

$$
\left|f_{n}\left(\lambda_{n} x_{n}+\mu_{n} y_{n}\right)\right| \geq\left|f_{n}\left(\lambda_{n} x_{n}+\mu_{n} y_{n}\right)-f_{n}\left(\lambda_{n} x_{n}\right)\right|-\left|f_{n}\left(\lambda_{n} x_{n}\right)\right|>n
$$

for every $n$. Since $\left(\mu_{n} y_{n}\right)_{n}$ tends to zero and $\left\|\lambda_{n} x_{n}\right\| \leq 1 / n$, the sequence $\left(\lambda_{n} x_{n}+\mu_{n} y_{n}\right)_{n}$ is bounded. Hence the sequence $\left(f_{n}\right)_{n} \subset B$ is not bounded on bounded sets in contradiction with the $\tau_{b}$-boundedness of $B$.

Several examples of locally convex Q-reflexive spaces are given in [4]. We will pay special attention to one of them, $\prod_{k=1}^{\infty} T_{J}{ }^{*}$, where $T_{J}{ }^{*}$ denotes the Tsirelson-James space.

EXAmple 4.11. Let $E:=\prod_{k=1}^{\infty} T_{J}{ }^{*}$. We will show that the spaces $\overline{\left(\mathscr{H}_{b}(E), \tau_{b}\right)_{\beta}^{\prime}}$ and $G_{b}\left(E_{\beta \beta}^{\prime \prime}\right)$ are isomorphic.

The space $E:=\prod_{k=1}^{\infty} T_{J}{ }^{*}$ is a Fréchet space (moreover, a quojection), hence the $\tau_{b}$-bounded sets of $\mathscr{P}\left({ }^{n} E_{\beta \beta}^{\prime \prime}\right)$ are locally bounded. By ([4], Example 3) $E$ is Q-reflexive. According to Proposition 4.9 it suffices to show that conditions (A) and (B) of Proposition 2.1 hold.

Let $B$ be a bounded subset of $\left(H_{b}(E), \tau_{b}\right)$. By ([11], Theorem 1.5$)$, for every $f \in \mathscr{H}_{b}(E)$ there exists a function $A B(f)$ in $\mathscr{H}_{b}\left(E_{\beta \beta}^{\prime \prime}\right)$ such that $\left.A B(f)\right|_{E}=f$. Let

$$
B^{\prime \prime}:=\{A B(f): f \in B\}
$$

and let $A^{\prime \prime}$ be a bounded subset of $E_{\beta \beta}^{\prime \prime}$. Since $E$ is a distinguished Fréchet space there exists a bounded subset of $E, A$, such that $A^{\prime \prime} \subset A^{\circ \circ}$. Using ([11], Theorem 1.5) we get

$$
\sup _{\tilde{f} \in B^{\prime \prime}}\|\tilde{f}\|_{A^{\prime \prime}}=\sup _{f \in B}\|A B(f)\|_{A^{\prime \prime}} \leq \sup _{f \in B}\|A B(f)\|_{A^{\circ \circ}}=\sup _{f \in B}\|f\|_{A}<\infty .
$$

Consequently the set $B^{\prime \prime}$ is $\tau_{b}$-bounded in $\mathscr{H}_{b}\left(E_{\beta \beta}^{\prime \prime}\right)$ and $\sup \left\{|\varphi(f)|: f \in B^{\prime \prime}\right\}$ is a continuous semi-norm on $G_{b}\left(E_{\beta \beta}^{\prime \prime}\right)$. Let $\vartheta=\sum_{n=0}^{\infty} \vartheta_{n} \in G_{b}\left(E_{\beta \beta}^{\prime \prime}\right)$. Since 
$E_{\beta \beta}^{\prime \prime}$ is Fréchet, every $\vartheta_{n}$ has a representation $\vartheta_{n}=\sum_{i=1}^{\infty} \lambda_{n}^{i} \otimes_{n} x_{n}^{i}$ for some null sequence $\left(x_{n}^{i}\right)_{i} \subset E_{\beta \beta}^{\prime \prime}$ and some $\left(\lambda_{n}^{i}\right)_{i} \in \ell_{1}$. Then

$$
\begin{aligned}
\sup _{f \in B}\left|\left[J_{n}\left(\vartheta_{n}\right)\right]\left(\frac{\widehat{d}^{n} f(0)}{n !}\right)\right| & =\sup _{f \in B}\left|\sum_{i=1}^{\infty} \lambda_{n}^{i}\left[A B_{n}\left(\frac{\widehat{d}^{n} f(0)}{n !}\right)\right]\left(x_{n}^{i}\right)\right| \\
& =\sup _{\tilde{f} \in B^{\prime \prime}}\left|\vartheta_{n}\left(\frac{\widehat{d^{n}} \tilde{f}(0)}{n !}\right)\right| .
\end{aligned}
$$

Hence condition (A) of Proposition 2.1 is satisfied.

Let $B^{\prime \prime}$ be a bounded subset of $\left(\mathscr{H}_{b}\left(E_{\beta \beta}^{\prime \prime}\right), \tau_{b}\right)$. By Lemma 4.10 there exists $n_{0} \in \mathrm{N}$ such that $f(x+y)=f(x)$ for all $f \in B^{\prime \prime}, x \in E_{n_{0}}^{\prime \prime}$ and $y \in$ $\left(E^{n_{0}}\right)^{\prime \prime}:=\prod_{j=n_{0}+1}^{\infty}\left(T_{J}{ }^{*}\right)^{\prime \prime}$. The space $E_{n_{0}}=\underbrace{T_{J}{ }^{*} \times \cdots \times T_{J}{ }^{*}}_{n_{0}}$ is a Q-reflexive Banach space, and since $E_{n_{0}}^{\prime \prime}$ has the RNP, $E_{n_{0}}$ is isometrically Q-reflexive ([8], Proposition 2.48). By ([9], Proposition 2) $\left(\mathscr{H}_{b}\left(E_{n_{0}}\right), \tau_{b}\right)^{\prime \prime}=\mathscr{H}_{b}\left(E_{n_{0}}^{\prime \prime}\right)$, and hence the set $J^{*}\left(B^{\prime \prime}\right)$ is contained and bounded in $\left(\mathscr{H}_{b}\left(E_{n_{0}}\right), \tau_{b}\right)^{\prime \prime}$. Since the spaces $\left\{\mathscr{P}\left({ }^{n} E_{n_{0}}\right)\right\}_{n}$ are Banach, by ([1], Proposition 8) $\left(H_{b}\left(E_{n_{0}}\right), \tau_{b}\right)$ is a quasinormable and consequently distinguished Fréchet space. Hence there exists a $\tau_{b}$-bounded set $B$ in $\mathscr{H}_{b}\left(E_{n_{0}}\right)$ such that $B^{\prime \prime} \subset B^{\circ 0}$. Since $E_{n_{0}}$ is isometrically Q-reflexive $\left\|J_{n}^{-1}\right\|=1$ for every $n$. Thus

$$
\begin{aligned}
\sup _{f \in B^{\prime \prime}}\left|\left[J_{n}^{-1}\left(\varphi_{n}\right)\right]\left(\frac{\widehat{d}^{n} f(0)}{n !}\right)\right| & \leq \sup _{f \in B^{\circ \circ}}\left|\left[J_{n}^{-1}\left(\varphi_{n}\right)\right]\left(\frac{\widehat{d^{n}} f(0)}{n !}\right)\right| \\
& =\sup _{f \in B}\left|\varphi_{n}\left(\frac{\widehat{d^{n}} f(0)}{n !}\right)\right| .
\end{aligned}
$$

The set $B$ is $\tau_{b}$-bounded in $\mathscr{H}_{b}\left(E_{n_{0}}\right)$ and hence in $\mathscr{H}_{b}(E)$. Thus (B) of Proposition 2.1 is satisfied.

AcKNOWLEDGements. I would like to thank Professor Seán Dineen for his remarks and suggestions.

\section{REFERENCES}

1. Ansemil, J., On the quasinormability of $H_{b}(U)$, Extracta Math. 9 (1994), no. 1, 71-74.

2. Bierstedt, K.-D., and Bonet, J., Biduality in Fréchet and (LB)-spaces, in Progress in Functional Analysis, Ed. K. Bierstedt et al., North-Holland Math. Stud. 170 (1992), 113-133.

3. Boyd, C., Distinguished preduals of spaces of holomorphic functions, Rev. Math. Univ. Complut. Madrid 6 (1993), no. 2, 221-231. 
4. Boyd, C., Dineen, S., and Venkova, M., Q-reflexive locally convex spaces, Publ. Res. Inst. Math. Sci. 40 (2004), 7-27.

5. Boyd, C., and Ryan, R., Geometric theory of spaces of integral polynomials and symmetric tensor products, J. Funct. Anal. 179 (2001), 18-42.

6. Dimant, V., Galindo, P., Maestre, M., and Zalduendo, I., Integral holomorphic functions, Studia Math. 160 (2004), no. 1, 83-99.

7. Dineen, S., Complex Analysis in Locally Convex Spaces, North-Holland Math. Stud. 57 (1981).

8. Dineen, S., Complex Analysis on Infinite Dimensional Spaces Monogr. Math., 1999.

9. Dineen, S., Canonical mappings for polynomials and holomorphic functions on Banach spaces, Proc. Amer. Math. Soc. 129 (2001), no. 10, 2897-2905.

10. Galindo, P., García, D., and Maestre, M., Holomorphic mappings of bounded type on (DF)spaces, Progress in Functional Analysis, Ed. K. Bierstedt et al., North-Holland Math. Stud. 170 (1992), 135-148.

11. Galindo, P., García, D., and Maestre, M., Entire functions of bounded type on Fréchet spaces, Math. Nachr. 161 (1993), 185-198.

12. Galindo, P., Maestre, M., and Rueda, P., Biduality in spaces of holomorphic functions, Math. Scand. 86 (2000), 5-16.

13. Horváth, J., Topological Vector Spaces and Distributions, vol. I, Addison-Wesley, Reading Massachusetts, 1966.

14. Jarchow, H., Locally Convex Spaces, B.G. Teubner, Stuttgart, 1981.

15. Mujica, J., and Nachbin, L., Linearization of holomorphic mappings on locally convex spaces, J. Math. Pures Appl. 71 (1992), 543-560.

SCHOOL OF MATHEMATICAL SCIENCES

UNIVERSITY COLLEGE DUBLIN

BELFIELD, DUBLIN 4

IRELAND

E-mail: Milena.Venkova@ucd.ie 